\title{
BREASTFEEDING PROBLEMS DURING FIRST THREE POSTNATAL DAYS
}

\author{
J. Raju ${ }^{1}$, M. Vatsa ${ }^{1}$, R.K. Agarwal ${ }^{2}$ \\ ${ }^{1}$ College of Nursing, ${ }^{2}$ Department of Pediatrics, All India Institute of Medical Sciences, New Delhi, India
}

Background and aims: We surveyed late preterm and term mother-newborn dyads $(n=380)$ in a tertiary care hospital to determine the epidemiology of breastfeeding problems during initial 72 hours.

Methods: The subjects were enrolled within 12 hours of delivery and assessed 12 hourly for perception of and documented breastfeeding problems using validated open ended questionnaire and checklist respectively.

Results: Most mothers had vaginal delivery $(220 ; 57.9 \%)$, no previous experience of breastfeeding (228; $60.0 \%)$, were graduates $(170 ; 44.7 \%)$ and received antenatal care $(100 \%)$.

All mothers had one or more perceived or documented breastfeeding problem. Perceived breastfeeding problems included insufficiency of milk $(89.7 \%, 95 \%$ CI 86.7 to 92.8$)$, difficulty in latching $(38.2 \%, 95 \%$ CI 33.3 to 43.0$)$, breast and nipple related problems $(29.5 \%, 95 \%$ CI 24.9 to 34.1$)$.

Documented breastfeeding problems were poor positioning and attachment (99.5\%, 95\% CI 98.7 to 100.0$)$, engorgement $(20 \%, 95 \%$ CI 16.0 to 24.0$)$, flat/inverted nipple $(11.3 \%, 95 \%$ CI 8.1 to 14.5$)$, sore nipple $(6.6 \%, 95 \%$ CI 4.1 to 9.0$)$ and cracked nipple (3.2\%, 95\% CI 1.4 to 4.9$)$.

Predictors of documented breastfeeding problems were education up to senior secondary (OR 3.8; 95\% CI $1.0,14.7)$ and having a large for date baby (OR $2.5 ; 95 \%$ CI $1.0,6.1)$.

Conclusion: There is a large burden of breastfeeding problems in mothers delivering at or near term. These findings have a major policy implication for planning postnatal care of mothers and babies. 